\title{
Dislocation of the temporomandibular joint following general anesthesia
}

\author{
Insoo Han, Tae Kwane Kim, Joo-Hyun Yoo, Jae Hong Park, and Eun Yong Chung \\ Department of Anesthesiology and Pain Medicine, Bucheon St. Mary's Hospital, The Catholic University of Korea College of Medicine, \\ Bucheon, Korea
}

Anesthesiologists are often concerned with the immobile temporomandibular joint (TMJ), but are sometimes faced with hypermobility and subluxation of the TMJ. Such TMJ instability has a prevalence of up to $25-50 \%$ in the general population, and is most common in middle-aged females [1]. TMJ dislocation that occurs during operations is often unnoticed until patients become aware of pain and swelling. Unrecognized TMJ displacement can lead to chronic pain and restricted joint mobility [2]. We report here two patients who had TMJ dislocation; one was observed in the post-anesthetic care unit (PACU) and the other in the operation room before transfer to the PACU.

A 34-year-old man weighing $78 \mathrm{~kg}$ was scheduled to undergo laparoscopic appendectomy. General anesthesia was conducted uneventfully and the duration of anesthesia was 1 hour. During the following 30 minutes in the PACU, a nurse noticed that the patient's mouth remained wide open. The patient complained of pain over the left parotid region, and mentioned he had experienced TMJ dislocation before. The diagnosis of anterior left TMJ dislocation was made and a manual reduction of TMJ was easily performed by a dentist.

An 18-year-old woman weighing $79 \mathrm{~kg}$ underwent tonsillectomy; she did not have a history of prior TMJ dysfunction. General anesthesia was done uneventfully and the duration of anesthesia was 90 minutes. When oropharynx was suctioned prior to extubation, the patient's mouth was widely opened and remained open. Diagnosis of dislocation of the mandible was confirmed and immediately corrected by the dentist.

TMJ dislocation is the dislodgement of the condyle from its normal position in the squamo-temporal portion of the cranial base [2,3]. It could occur spontaneously or by trauma, endotracheal intubation, LMA insert, ENT/dental procedures, endoscopy, yawning, laughing, vomiting, transesophageal echo probe placement, seizure, congenital anomaly and medication [4]. Acute TMJ dislocation is associated with pain and severe tenderness over the TMJ areas. If left untreated for longer than 14 days, fibrosis and even fractures become increasingly apparent, which may require surgical treatment $[3,4]$.

TMJ disorders can affect airway management during surgery. If the patient has a history of TMJ dislocation, we should take more caution during airway management. We should also provide information regarding the possibility of postoperative TMJ dislocation, because patients cannot comprehend a TMJ dislocation at a non-face surgery. Argo et al. [5] reported increased symptoms following endotracheal intubation in $44 \%$ of patients with preexisting TMJ dysfunction.

At the recovery period, we should be concerned about patients who complain pain around the TMJ area, particularly patients with a history of TMJ disorders and who cannot speak well. If the patient is unconscious or unable to respond, a physical examination by checking the range of motion of the TMJ would help [2,4].

In conclusion, TMJ dislocation is a common side effect of airway manipulation, particularly in a sedated or anesthetized patient. However, its diagnosis can easily be overlooked. For prevention, manipulation involving the upper airway should be firm but gentle. Also, early recognition and treatment of TMJ dislocation would reduce the incidence of long-term complications.

Corresponding author: Eun Yong Chung, M.D., Ph.D., Department of Anesthesiology and Pain Medicine, Bucheon St. Mary's Hospital, The Catholic University of Korea College of Medicine, 327, Sosa-ro, Wonmi-gu, Bucheon 420-717, Korea. Tel: 82-32-340-2158, Fax: 82-32-340-2255, E-mail: anes36@catholic.ac.kr

() This is an open-access article distributed under the terms of the Creative Commons Attribution Non-Commercial License (http:// creativecommons.org/licenses/by-nc/3.0/), which permits unrestricted non-commercial use, distribution, and reproduction in any medium, provided the original work is properly cited. 


\section{References}

1. Awsare AN, Prakash N. Temporomandibular dislocation: should every doctor be trained in resetting the jaw? Br J Oral Maxillofac Surg 2006; 44: 339.

2. Roze des Ordons A, Townsend DR. Trachlight management of succinycholine-induced subluxation of the temporo-mandibular joint: a case report and review of the literature. Can J Anaesth 2008; 55: 616-21.

3. Akinbami BO. Evaluation of the mechanism and principles of management of temporomandibular joint dislocation. Systematic review of literature and a proposed new classification of temporomandibular joint dislocation. Head Face Med 2011; 7: 10.

4. Pillai S, Konia MR. Unrecognized bilateral temporomandibular joint dislocation after general anesthesia with a delay in diagnosis and management: a case report. J Med Case Rep 2013; 7: 243.

5. Argo F, Salvinelli F, Casales M, Antonelli S. Temporomandibular joint assessment in anaesthetic practice. Br J Anaesth 2003; 90: 707-8. 